\title{
The problem of atrial arrhythmias after noncardiac thoracic surgery
}

Lawrence L. Creswell, MD

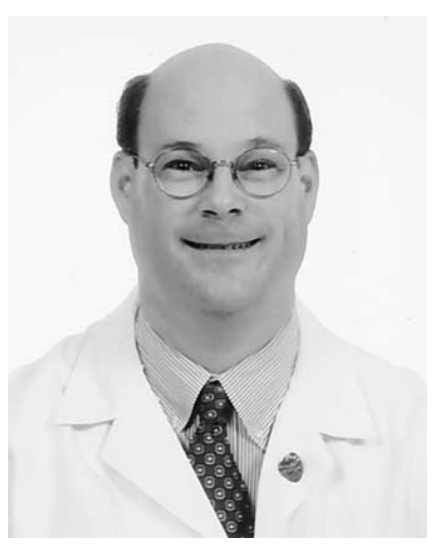

Dr Creswell

See related article on page 779.
From the University of Mississippi Medical Center, Jackson, Miss.

Received for publication Sept 30, 2003; accepted for publication Oct 6, 2003.

Address for reprints: Lawrence L. Creswell, MD, University of Mississippi Medical Center, 2500 North State St, Jackson, MS 39216 (E-mail: 1creswell@ surgery. umsmed.edu).

J Thorac Cardiovasc Surg 2004;127:629-30 $0022-5223 / \$ 30.00$

Copyright () 2004 by The American Association for Thoracic Surgery

doi:10.1016/j.jtcvs.2003.10.013

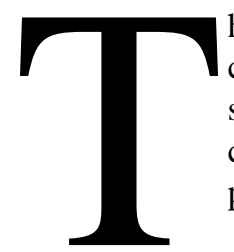

he problem of postoperative atrial fibrillation (AF) has received considerable attention over the years. Nonetheless, this is probably still the most common complication in adult patients undergoing cardiac surgery and one of the most common complications in adult patients undergoing noncardiac thoracic surgery.

Perhaps because of effective antiarrhythmic medications that can be used to treat patients with postoperative AF, these arrhythmias were often thought to be benign, producing only transient discomfort, palpitations, or shortness of breath. For patients undergoing cardiac surgery, we now have substantial evidence that postoperative AF is associated with increases in the use of hospital resources, length of hospital stay, and hospital costs. Moreover, postoperative AF has been associated with a variety of other adverse outcomes, including perioperative myocardial infarction, congestive heart failure (CHF), ventricular arrhythmias, renal insufficiency, infection, pneumonia, increased need for inotropic medications, increased use of the intra-aortic balloon pump, prolonged ventilation, increased frequency of reintubation for respiratory distress, increased need for readmission to the intensive care unit, and increased need for tracheostomy. There is a growing recognition that one of the most important clinical consequences of postoperative AF might be an increased incidence of perioperative stroke. We have also come to recognize that these arrhythmias might be persistent or recurrent, even long after discharge from the hospital.

Clinical investigation in adults undergoing cardiac surgery has focused mainly on 2 fronts: (1) the identification of risk factors for the development of postoperative $\mathrm{AF}$ and (2) various strategies for prophylaxis. The most commonly identified risk factors in retrospective studies are a previous history of atrial arrhythmias and increasing patient age. Other risk factors that have been reported include valvular heart disease, male sex, chronic obstructive pulmonary disease, the presence of right coronary artery stenosis, left ventricular hypertrophy, CHF, and various electrolyte abnormalities.

Many investigators have suggested that patients who could be identified at high risk for this complication prospectively might benefit from prophylaxis strategies. It is important to remember that because postoperative AF occurs in only the minority of patients, many patients must be treated to prevent this complication in just the minority. As a result, prophylaxis strategies should come at low risk and (relatively) low cost. Prospective randomized trials have examined the utility of a variety pharmacologic agents used prophylactically, including $\beta$-blockers, sotalol, amiodarone, verapamil, diltiazem, magnesium, digoxin, dexamethasone, glucose-insulinpotassium infusions, tri-iodothyronine, procainamide, alinidine, and quinidine. The results have often been conflicting, and as a result, there is no consensus. Most authorities would agree that the use of $\beta$-blockers postoperatively can serve as effective prophylaxis. The evidence also suggests that amiodarone or sotalol, when used appropriately, can reduce the incidence of postoperative AF in adults undergoing cardiac surgery. Evidence from prospective randomized trials also suggests that the use of mild (rather than moderate) hyothermia, posterior pericardiotomy, atrial pacing, and heparin-coated cardiopulmonary bypass circuits might help to reduce the incidence of postoperative AF. 
The issue of postoperative AF after noncardiac surgery has received much less attention. Except for patients undergoing major thoracic, vascular, and abdominal procedures, the incidence of postoperative AF is probably very low. The study of adult patients undergoing noncardiac thoracic surgery has been limited somewhat because of the heterogeneous patient population and because, except for a few centers, single institutions do not have large (eg, hundreds of cases per year) patient populations to analyze. As a result, most reports have focused on small numbers of patients undergoing pulmonary resection. There seems to be good evidence that the extent of pulmonary resection is associated with a differential incidence of postoperative AF, with more arrhythmias after pneumonectomy than after lobectomy. There have been reports of pharmacologic prophylaxis with digoxin, flecainide, verapamil, $\beta$-blockers, amiodarone, and diltiazem, but these studies have included small numbers of patients and produced mixed results. As a result, in most thoracic surgery practices today, most patients probably receive no pharmacologic prophylaxis for postoperative AF.

Vaporciyan and colleagues, ${ }^{1}$ elsewhere in this issue of the Journal, report on a very detailed analysis of 2588 patients undergoing noncardiac thoracic surgical procedures, focusing on the incidence and risk factors for postoperative AF. These investigators report an overall incidence of postoperative AF of $12.3 \%$, and this is in keeping with the results of previous reports. We learn from this report that significant multivariate predictors of postoperative AF include male sex, increasing patient age, history of $\mathrm{CHF}$, history of arrhythmias, and history of peripheral vascular disease. We also learn that certain types of noncardiac thoracic operations are associated with an increased risk of postoperative AF, including resection of mediastinal tumor or thymectomy, lobectomy, bilobectomy, and pneumonectomy. In keeping with previous reports, more extensive pulmonary resection (eg, pneumonectomy) was associated with more postoperative AF than limited pulmonary resection (eg, lobectomy). Interestingly, the authors report that intraoperative blood transfusion was a risk factor for postoperative AF.

How should we use the information at hand about postoperative AF after noncardiac thoracic surgery? Drawing on our experience with patients undergoing cardiac surgery, it is probably prudent to avoid $\beta$-blocker withdrawal if possible and to use $\beta$-blockers postoperatively in patients who can tolerate these medications. We should also try to iden- tify patients at high risk for postoperative AF who might be appropriate candidates for prophylaxis. In this subset of patients, it might be reasonable to consider the use of agents such as amiodarone preoperatively and postoperatively to try to prevent the development of postoperative AF.

Many important questions remain. The natural history of postoperative AF after noncardiac surgery should be defined. Are these arrhythmias, whether treated or not, selflimiting? What is the rate of persistent or recurrent AF in this patient population, and how does this affect the patient's care? In the setting of cardiac surgery, it is widely believed that antiarrhythmic medications can be discontinued at 2 to 3 months after the operation, but is the same true for patients undergoing noncardiac thoracic surgery? Largescale trials of pharmacologic prophylaxis and intraoperative strategies are needed, and these will probably need to be multi-institutional to accrue sufficient numbers of patients. These are the sorts of trials that might be supported by our professional organizations, such as the American College of Surgeons, the American Association for Thoracic Surgery, or the Society of Thoracic Surgeons. At this point, amiodarone administered orally for several days before the operation and then continued postoperatively is probably the most attractive option. Unlike in cardiac surgery, many noncardiac thoracic surgery procedures are elective, allowing for a week or more of pharmacologic therapy before the operation. It is obvious that we must have larger trials of potential pharmacologic prophylaxis strategies, but we must also test whether the administration of any prophylactic agent results in not only fewer arrhythmias but also, for example, reduces costs and decreases the length of hospital stay. The use of intraoperative and perioperative strategies, such as thoracic epidural anesthesia and atrial pacing, should also be tested in these patients for efficacy. Lastly, a broader question should be answered: Is anticoagulation with rate control as effective as antiarrhythmic therapy for these patients?

The article by Vaporciyan and colleagues ${ }^{1}$ reminds us that the issue of postoperative AF after noncardiac thoracic surgery is an important one. We should learn more and do more for our patients undergoing noncardiac thoracic surgery to prevent this complication.

\section{Reference}

1. Vaporciyan AA, Correa AM, Rice DC, Roth JA, Smythe WR, Swisher SG, et al. Risk factors associated with atrial fibrillation after noncardiac thoracic surgery: analysis of 2588 patients. J Thorac Cardiovasc Surg. 2004;127:779-86 